\title{
Principales conclusions émanant d'un système national de surveillance amélioré du VIH : 2010-2012
}

\author{
Tarasuk $\mathrm{J}^{1 *}$, Ogunnaike-Cooke $\mathrm{S}^{1}$, Archibald $\mathrm{C}^{1}$, MacLean $\mathrm{R}^{1}$, Bennett $\mathbf{R}^{1}$, Kim $\mathrm{J}^{1}$, Malloch $\mathrm{L}^{1}$ \\ au nom des chercheurs principaux de I-Track
}

\footnotetext{
${ }^{1}$ Centre de la lutte contre les maladies transmissibles et les infections, Agence de la santé publique du Canada, Ottawa (Ontario) ${ }^{*}$ Correspondance: jill.tarasuk@phac-aspc.gc.ca
}

\section{Résumé}

Contexte : Les utilisateurs de drogues injectables représentent un groupe à risque important dans l'épidémie de VIH au Canada. I-Track est un système de surveillance national de santé publique conçu pour surveiller la prévalence du VIH et de l'hépatite $\mathrm{C}$ ainsi que les facteurs comportementaux à risque associés parmi les utilisateurs de drogues injectables au Canada. L'information est recueillie grâce à des enquêtes transversales menées périodiquement dans des sites sentinelles dans tout le Canada. La phase 3 de I-Track a été réalisée entre le 26 avril 2010 et le 7 août 2012 dans 11 sites sentinelles participants.

Objectif : Évaluer la prévalence du VIH, l'exposition à vie à l'hépatite $\mathrm{C}$ et les comportements à risque associés chez les utilisateurs de drogues injectables au Canada afin d'orienter les activités de prévention, de traitement et de contrôle du VIH et de l'hépatite $C$ et d'en faciliter l'évaluation.

Méthodologie : Des personnes s'étant injecté des drogues dans les six mois précédant l'entrevue et répondant aux exigences minimales relatives à l'âge du consentement ont participé à une enquête administrée par un interviewer et ont fourni un échantillon de sang aux fins de dépistage des anticorps contre le VIH et l'hépatite C. Des analyses descriptives ont été effectuées par le biais de comparaisons en fonction du sexe.

Résultats : 2687 personnes ont participé à cette enquête. De ces participants, $68,2 \%$ étaient de sexe masculin, 60,9 \% étaient âgés de 30 à 49 ans et $36,2 \%$ s'étaient déclarés Autochtones. Parmi les participants qui ont fourni un échantillon de sang suffisant aux fins du dépistage, $11,2 \%$ ont été trouvés séropositifs pour le VIH et leur exposition à vie à l'infection par l'hépatite C était de $68 \%$. Parmi les drogues généralement injectées, on trouve la cocaïne $(64,3 \%)$, l'hydromorphone $(47,2 \%)$, la morphine non prescrite $(47 \%)$, l'oxycodone $(37,7 \%)$ et l'héroïne $(26,7 \%)$. Les participants ont déclaré s'injecter des drogues avec des aiguilles (15,5\%) ou autre équipement d'injection $(34,5 \%)$ déjà utilisés. Un peu plus d'un tiers $(34,4 \%)$ des participants ont déclaré avoir eu deux partenaires sexuels ou plus dans les six mois précédant l'entrevue et $36,6 \%$ ont signalé avoir utilisé un condom lors de leur dernière relation sexuelle. La majorité des participants avaient subi un test de dépistage du VIH ou de l'hépatite $\mathrm{C}$ au moins une fois dans leur vie $(92,9 \%$ et $91,4 \%$, respectivement). Une grande proportion des participants qui avaient déclaré être séropositifs était suivie par un médecin $(95 \%)$ et près des deux tiers d'entre eux prenaient des médicaments prescrits pour leur infection au VIH au moment de l'entrevue (66 \%).

Conclusion : La séroprévalence du VIH et l'exposition à vie à l'infection par l'hépatite C étaient élevées parmi les participants à la phase 3 de I-Track. Bien que de nombreux participants aient déclaré adopter des pratiques d'injection et sexuelles sécuritaires, une proportion élevée de participants a déclaré avoir des comportements à risque associés à l'acquisition et à la transmission du VIH et de l'hépatite C. Les utilisateurs de drogues injectables représentent encore un groupe à risque important dans l'épidémie de $\mathrm{VIH}$ au Canada, et les résultats de la phase 3 du système I-Track mettent en évidence la nécessité de poursuivre les services de prévention et de traitement, ainsi que le dépistage régulier et intégré des utilisateurs de drogues injectables. 


\section{Introduction}

Certains comportements à risque observés chez les utilisateurs de drogues injectables, tels que le partage d'aiguilles et de tout autre matériel d'injection et les relations sexuelles non protégées, contribuent à la transmission des infections transmissibles par le sang, y compris le VIH et l'hépatite C. On estime qu'en 2011, les utilisateurs de drogues injectables représentaient $13,7 \%$ des cas de nouvelles infections au VIH et $16,9 \%$ des personnes vivant déjà avec le VIH au Canada (1). Les cas d'infection à l'hépatite $C$ chez les utilisateurs de drogues injectables au Canada continuent de représenter une préoccupation majeure en matière de santé publique; en effet, chaque année, l'utilisation de drogues injectables représente $61 \%$ des nouveaux cas d'infection à l'hépatite C au Canada (2).

Un système national de surveillance comportementale et biologique, nommé I-Track, a été conçu pour surveiller la prévalence du $\mathrm{VIH}$ et de l'hépatite $\mathrm{C}$ ainsi que les comportements à risque connexes parmi les populations autochtones qui utilisent des drogues injectables au Canada. Le projet pilote I-Track a été mené entre 2002 et 2003 dans quatre sites, suivi par trois phases de collecte de données : la phase 1 de 2003 à 2005 dans sept sites, la phase 2 de 2005 à 2008 dans 10 sites et la phase 3 de 2010 à 2012 dans 11 sites.

La surveillance continue des comportements à risque chez les personnes qui s'injectent des drogues fait office de système d'alerte rapide pour la propagation des infections transmissibles par le sang au Canada. En outre, les résultats de l'enquête I-Track peuvent aider à documenter et à évaluer les interventions existantes en matière de santé publique à l'égard du VIH et de l'hépatite $C$ chez les personnes qui s'injectent des drogues au Canada.

Le présent rapport contient certains résultats de la phase 3 de l-Track et est un résumé d'un rapport plus approfondi (3).

\section{Méthodologie}

Le système I-Track est un système de surveillance comportementale et biologique qui contrôle la prévalence du VIH et de l'hépatite $C$ ainsi que les comportements à risque connexes parmi les populations autochtones qui utilisent des drogues injectables au Canada. L'information est recueillie grâce à des enquêtes transversales menées périodiquement dans des sites sentinelles dans tout le Canada. Les enquêtes de la phase 3 de I-Track ont été réalisées entre le 26 avril 2010 et le 7 août 2012 dans 11 sites sentinelles participants.

La population cible était constituée de personnes ayant utilisé des drogues injectables au cours des six mois précédant le recrutement et qui répondaient aux exigences minimales relatives à l'âge du consentement conformément aux exigences provinciales. La participation était volontaire, totalement anonyme et fondée sur un consentement verbal éclairé. Les participants consentants étaient invités à répondre à un questionnaire remis par un intervieweur couvrant les caractéristiques démographiques, l'usage de substances psychoactives et les comportements d'injection, les comportements sexuels, le dépistage du VIH et de l'hépatite $\mathrm{C}$ et les antécédents de traitement, l'utilisation de services de santé, et les connaissances sur le VIH. Les participants étaient également invités à fournir un échantillon biologique faisant l'objet d'un test de dépistage des anticorps du VIH et de l'hépatite C. On a d'abord procédé au dépistage du VIH, puis au dépistage de l'hépatite $C$, si le volume de l'échantillon était suffisant.

Les données comprises dans ce rapport constituent les résultats descriptifs présentés pour l'échantillon global, ainsi que par sexe, afin de permettre les comparaisons entre les participants de sexe masculin et de sexe féminin en ce qui a trait aux caractéristiques démographiques, aux comportements d'usage et d'injection de drogues, aux comportements sexuels à risque, au dépistage, aux soins et au traitement liés au VIH et à l'hépatite $\mathrm{C}$, ainsi qu'à l'utilisation des services de santé. Les résultats concernant les connaissances sur le VIH ne sont pas présentés ici, mais peuvent être obtenus dans le rapport complet (3). Les résultats provenant des données des tableaux dont les cellules comportent de faibles nombres doivent être interprétés avec prudence. 


\section{Résultats}

Au total, 2687 personnes ont participé à la phase 3 de l-Track dans 11 sites sentinelles au Canada : Whitehorse (Yukon) $(n=55)$, Prince George (Colombie-Britannique) $(n=150)$, Edmonton (Alberta) $(n=183)$, Regina (Saskatchewan) $(n=$ 251), Thunder Bay (Ontario) $(n=138)$, Sudbury (Ontario) $(n=148)$, London (Ontario) $(n=204)$, Toronto (Ontario) $(n=$ 260 ), Kingston (Ontario) $(n=200)$, le réseau SurvUDI (sites de la province du Québec ${ }^{*}$ et à Ottawa [Ontario]) $(n=937)$ et Halifax (Nouvelle-Écosse) $(n=161)$. Les sites du réseau SurvUDI de la province du Québec comprennent : Abitibi-

Témiscamingue, Outaouais, Montréal, ville de Québec, Montérégie, Mauricie - Centre-du-Québec, Saguenay - Lac StJean et Cantons de l'Est.

Tableau 1 : Caractéristiques démographiques des participants à la phase 3 de I-TrackJ Tara

\begin{tabular}{|c|c|c|c|c|}
\hline $\begin{array}{l}\text { Caractéristiques démographiques et } \\
\text { antécédents d'incarcération }\end{array}$ & $\begin{array}{c}\text { Total }^{1} \\
(n=2687)\end{array}$ & $\begin{array}{l}\text { Hommes } \\
(n=1832)\end{array}$ & $\begin{array}{l}\text { Femmes } \\
(n=855)\end{array}$ & Valeur $p$ \\
\hline $\begin{array}{r}\text { Âge en nombre d'années ( } n=2687) \\
\text { Moins de } 30 \\
30 \text { à } 49 \\
50 \text { et plus }\end{array}$ & $\begin{array}{c}20,9 \%(561) \\
60,8 \% \\
(1635) \\
18,3 \%(491)\end{array}$ & $\begin{array}{c}16,8 \%(307) \\
62,3 \% \\
(1142) \\
20,9 \%(383)\end{array}$ & $\begin{array}{l}29,7 \%(254) \\
57,7 \%(493) \\
12,6 \%(108)\end{array}$ & $<0,001$ \\
\hline $\begin{array}{l}\text { Origine autochtone autodéclarée (Premières } \\
\text { nations, Métis ou Inuits) ( } n=2678)\end{array}$ & $36,2 \%(968)$ & $29,4 \%(537)$ & $50,6 \%(431)$ & $<0,001$ \\
\hline $\begin{array}{l}\text { Orientation sexuelle ( } n=2673 \text { ) } \\
\text { Hétérosexuel } \\
\text { Gai, lesbienne, bisexuel, bispirituel ou autre }\end{array}$ & $\begin{array}{c}88,3 \% \\
(2359) \\
11,7 \%(314) \\
\end{array}$ & $\begin{array}{c}91,9 \% \\
(1679) \\
8,1 \%(147) \\
\end{array}$ & $\begin{array}{l}80,3 \%(680) \\
19,7 \%(167)\end{array}$ & $<0,001$ \\
\hline $\begin{array}{r}\text { Niveau de scolarité ( } \mathrm{n}=\mathbf{2} 679) \\
\text { Quelques années d'études secondaires ou } \\
\text { moins } \\
\text { Diplôme d'études secondaires } \\
\text { A réalisé des études supérieures }\end{array}$ & $\begin{array}{c}55,7 \% \\
(1492) \\
20,9 \%(560) \\
23,4 \%(627)\end{array}$ & $\begin{array}{l}53,4 \%(974) \\
22,1 \%(403) \\
24,5 \%(447)\end{array}$ & $\begin{array}{l}60,6 \%(518) \\
18,4 \%(157) \\
21,2 \%(180)\end{array}$ & 0,002 \\
\hline $\begin{array}{r}\text { Revenu mensuel }(n=2641)^{2} \\
\text { Moins de } 500 \$ \\
\text { Entre } 500 \$ \text { et } 999 \$ \\
\text { Entre } 1000 \$ \text { et } 1999 \$ \\
2000 \$ \text { et plus }\end{array}$ & $\begin{array}{c}14,4 \%(379) \\
39,7 \% \\
(1049) \\
29,3 \%(775) \\
16,6 \%(438)\end{array}$ & $\begin{array}{l}12,3 \%(222) \\
40,3 \%(726) \\
28,8 \%(518) \\
18,6 \%(334)\end{array}$ & $\begin{array}{l}18,7 \%(157) \\
38,4 \%(323) \\
30,6 \%(257) \\
12,4 \%(104)\end{array}$ & $<0,001$ \\
\hline 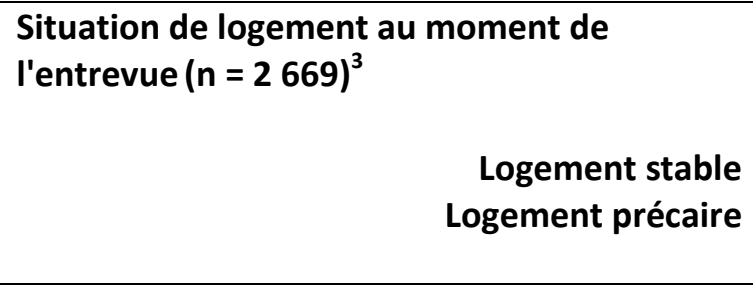 & $\begin{array}{l}61,3 \% \\
(1637) \\
38,7 \% \\
(1032)\end{array}$ & $\begin{array}{c}57,6 \% \\
(1049) \\
42,4 \%(772)\end{array}$ & $\begin{array}{l}69,3 \%(588) \\
30,7 \%(260)\end{array}$ & $<0,001$ \\
\hline $\begin{array}{l}\text { Proportion des participants ayant été } \\
\text { incarcérés au cours des six mois précédant } \\
\text { l'entrevue }(n=2683)^{4}\end{array}$ & $11,5 \%(308)$ & $12,5 \%(229)$ & $9,3 \%(79)$ & $<0,014$ \\
\hline $\begin{array}{l}\text { Proportion des participants ayant déjà vécu } \\
\text { dans un établissement correctionnel }\end{array}$ & $\begin{array}{l}82,5 \% \\
(2210)\end{array}$ & $\begin{array}{l}88,5 \% \\
(1618)\end{array}$ & $69,7 \%$ (592) & $<0,001$ \\
\hline
\end{tabular}


$(n=2678)$

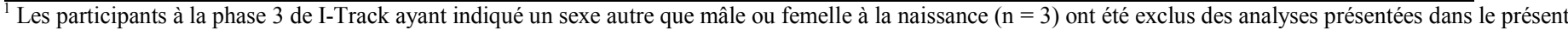
rapport.

${ }^{2}$ Cela incluait toutes les sources de revenus, légales et illégales, durant une période d'un mois.

${ }^{3}$ Les participants devaient indiquer l'endroit où ils vivaient au moment de l'entrevue et les réponses étaient classées en deux catégories : le logement stable ou le

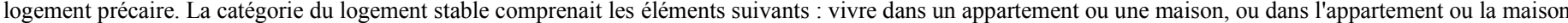

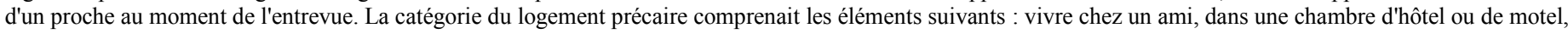

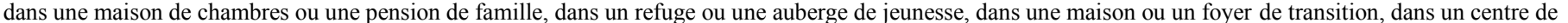

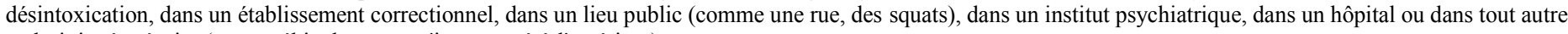
endroit jugé précaire (p. ex. véhicule, tente, n'importe où à l'extérieur).

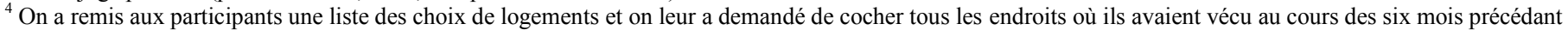
l'entrevue. Les participants ayant sélectionné un établissement correctionnel (prison ou service correctionnel) sont présentés ici.

Le tableau 1 indique que les caractéristiques sociodémographiques des participants à la phase 3 étaient semblables à celles des phases précédentes de I-Track; une grande proportion des participants était des hommes $(68,2 \%)$ et la plus grande proportion des participants étaient âgés de 30 à 49 ans (60,9\%), avec une proportion significativement plus élevée chez les hommes que chez les femmes de ce groupe d'âge (62,3\% par rapport à $57,7 \%)$ et dans le groupe d'âge des 50 ans et plus (20,9\% par rapport à 12,6\%). Bien que la majorité des participants à I-Track ait autodéclaré leur orientation sexuelle comme étant hétérosexuelle $(88,3 \%)$, une proportion beaucoup plus élevée de femmes que d'hommes s'est autodéfinie comme étant gaie, lesbienne, bisexuelle, bispirituelle ou autres (19,7\% par rapport à 8,1\%).

Plus d'un tiers (36,2\%) des participants s'étaient autodéclarés Autochtone (Premières nations, Métis ou Inuits), soit une proportion bien supérieure à la proportion d'Autochtones autodéclarés au sein de la population canadienne générale. D'après les données de 2011 issues de l'Enquête auprès des ménages, 4,3 \% de l'ensemble de la population du Canada s'identifient comme étant Autochtones (4). Il y avait un écart important entre l'ensemble des sites en ce qui concerne la proportion de participants s'étant déclarés d'origine autochtone. Par exemple, 89,6 \% des participants à Regina et $84,7 \%$ des participants à Edmonton se sont déclarés Autochtones, alors que seulement 19,1\% des participants à London et $13,7 \%$ des participants du réseau SurvUDI se sont déclarés Autochtones (données non représentées). En outre, une proportion significativement plus élevée de femmes dans tous les sites s'est déclarée Autochtone $(50,6 \%$ de femmes par rapport à 29,4\% d'hommes).

Plus de la moitié $(55,7 \%)$ des participants a déclaré avoir un niveau d'éducation inférieur aux études secondaires, avec une proportion significativement plus élevée de femmes ayant déclaré un niveau d'éducation inférieur par rapport à leurs homologues masculins. Plus d'un tiers $(39,7 \%)$ des participants ont indiqué que leur revenu mensuel se situait entre 500 \$ et $999 \$$, bien que l'on ait noté une variation considérable entre les participants et des différences importantes entre les hommes et les femmes.

Plus d'un tiers $(38,7 \%)$ de tous les participants a déclaré vivre dans un logement précaire au moment de l'entrevue, avec une proportion significativement plus élevée d'hommes ayant signalé vivre dans un logement précaire. Plus d'un dixième $(11,5 \%)$ a déclaré avoir vécu dans un établissement correctionnel au cours des six mois précédant l'entrevue; parmi les hommes, cette proportion (12,5\%) était nettement plus élevée que chez les femmes $(9,3 \%)$. Une grande proportion de tous les participants $(82,5 \%)$ ont indiqué qu'ils avaient, à un moment précis de leur vie, été incarcérés; la proportion d'hommes ayant déclaré des antécédents d'incarcération était significativement plus élevée que la proportion de femmes (88,5\% par rapport à 69,7\%). Un logement précaire et l'incarcération présentent des difficultés pour la prévention et le contrôle du VIH et d'autres infections transmissibles par le sang parmi les utilisateurs de drogues injectables au Canada, car ils représentent des environnements d'injection à un risque élevé $(5,6)$. 
Tableau 2 : Résultats en laboratoire du dépistage du VIH et de I'hépatite C des participants à l'enquête de la phase 3 de I-Track

\begin{tabular}{|c|c|c|c|c|}
\hline Résultats de laboratoire & Total & Hommes & Femmes & $\begin{array}{l}\text { Valeur } \\
p\end{array}$ \\
\hline \multicolumn{5}{|c|}{ Séroprévalence du VIH (parmi les participants ayant fourni un échantillon de sang, $n=2593)^{1}$} \\
\hline Participants séropositifs pour le VIH & $11,2 \%(291)$ & $11,6 \%(205)$ & $10,4 \%(86)$ & 0,387 \\
\hline $\begin{array}{r}\text { Proportion de participants } \\
\text { séropositifs pour le VIH qui étaient au } \\
\text { courant de leur séropositivité pour le VIH } \\
(\mathrm{n}=\mathbf{2 8 1})^{2}\end{array}$ & $78,6 \%(221)$ & $78,7 \%(155)$ & $78,6 \%(66)$ & 0,984 \\
\hline \multicolumn{5}{|c|}{ Exposition à vie à l'hépatite C (parmi les participants ayant fourni un échantillon de sang, $n=2575)^{3}$} \\
\hline Participants séropositifs pour l'hépatite $\mathrm{C}$ & $68 \%(1750)$ & $\begin{array}{l}67,9 \% \\
(1192)\end{array}$ & $68,1 \%(558)$ & 0,899 \\
\hline \multicolumn{5}{|c|}{$\begin{array}{l}\text { État sérologique pour le VIH et l'hépatite C (parmi les participants ayant fourni un échantillon de sang en } \\
\text { quantité suffisante pour effectuer les tests de dépistage d'anticorps contre le VIH et l'hépatite } C \\
(n=2575)\end{array}$} \\
\hline $\begin{array}{r}\text { Participants séropositifs pour le VIH } \\
\text { uniquement }^{1}\end{array}$ & $1,7 \%(43)$ & $2 \%(35)$ & $1 \%(8)$ & \multirow{4}{*}{0,312} \\
\hline $\begin{array}{r}\text { Participants séropositifs pour l'hépatite C } \\
\text { uniquement }{ }^{3}\end{array}$ & $\begin{array}{l}58,5 \% \\
(1505)\end{array}$ & $\begin{array}{l}58,4 \% \\
(1025)\end{array}$ & $58,6 \%(480)$ & \\
\hline $\begin{array}{r}\text { Participants séropositifs pour le VIH et } \\
{\text { l'hépatite } C^{1,3}}\end{array}$ & $9,5 \%(245)$ & $9,5 \%(167)$ & $9,5 \%(78)$ & \\
\hline $\begin{array}{r}\text { Participants séronégatifs pour le VIH et } \\
\text { l'hépatite C }\end{array}$ & $30,4 \%(782)$ & $30,1 \%(529)$ & $30,9 \%(253)$ & \\
\hline
\end{tabular}

${ }^{1}$ Le dépistage du VIH grâce à des échantillons de gouttes de sang séchées a été réalisé en utilisant la méthode AVIOQ HIV-1 EIA. Des tests de confirmation Western Blot ont ensuite été réalisés (Bio-Rad GS HIV-1). Un résultat positif indiquait une infection au VIH.

${ }^{2}$ Les participants qui ont indiqué que les derniers résultats de leur test de dépistage du VIH étaient positifs et qui ont été identifiés comme étant séropositifs pour le VIH d'après l'analyse de l'échantillon biologique fourni au moment de l'entrevue ont été classés comme étant au courant de leur séropositivité pour le VIH.

${ }^{3}$ Le dépistage de l'hépatite $\mathrm{C}$ grâce à des échantillons de sang séché a été réalisé en utilisant la version 3.0 du test VHC EIA Ortho ${ }^{\mathrm{MD}}$. Les tests de confirmation n'ont pas été effectués sur les échantillons dont le test de dépistage était positif. Un résultat positif indiquait une infection passée ou présente par l'hépatite $\mathrm{C}$, et ne permettait pas d'établir de distinction entre une infection aiguë, chronique ou résolue.

Dans l'ensemble, la séroprévalence du VIH et l'exposition à vie à l'infection par l'hépatite C étaient élevées (tableau 2). En effet, $11,2 \%$ des participants à l'enquête ayant fourni un échantillon biologique en quantité suffisante pour effectuer le test de dépistage étaient séropositifs pour le VIH ainsi et 68 \% étaient séropositifs pour l'hépatite $\mathrm{C}$. Aucune différence importante dans la séroprévalence du VIH et de l'hépatite C n'a été observée entre les hommes et les femmes. Bien qu'il soit impossible de déterminer la proportion de participants qui étaient infectés aussi bien par le VIH que par l'hépatite $C$ au moment de l'entrevue en raison de la nature de l'épreuve de laboratoire utilisée (c'est-à-dire qu'il n'a pas été possible d'établir la différence entre une infection présente et une infection passée par l'hépatite $C$ ), la proportion de participants qui étaient séropositifs pour le VIH et l'hépatite $C(9,5 \%)$ met toutefois en évidence la possibilité de multiples infections compliquant les réponses au traitement ainsi que les résultats en matière de santé parmi les utilisateurs de drogues injectables au Canada.

La nécessité du dépistage régulier et intégré du VIH et de l'hépatite $\mathrm{C}$ parmi les utilisateurs de drogues injectables ne peut être exagérée. On a découvert que seulement $78,6 \%$ des participants à la phase 3 de I-Track ayant obtenu un résultat positif au test de dépistage du VIH à partir d'échantillons biologiques fournis au moment de l'entrevue étaient conscients de leur infection, ou bien que $21,4 \%$ des participants séropositifs n'étaient pas au courant de leur séropositivité pour le VIH. Les personnes qui ne sont pas au courant de leur état sérologique ne peuvent pas bénéficier d'un traitement et de services de consultation et, en outre, ne peuvent pas prendre de mesures pour réduire leur risque de transmission du VIH à d'autres personnes. En outre, le dépistage permet d'accroître la sensibilisation aux pratiques 
sexuelles et d'injection sécuritaires parmi les utilisateurs de drogues injectables, et d'orienter les personnes vers les services de santé et de soutien social disponibles. 
Tableau 3 : Comportements d'usage et d'injection de drogues chez les participants à l'enquête de la phase 3 de I-Track

\begin{tabular}{|c|c|c|c|c|}
\hline Comportements d'usage de drogues & Total & Hommes & Femmes & Valeur $p$ \\
\hline $\begin{array}{l}\text { Proportion de participants dont la } \\
\text { première injection est survenue avant } \\
\text { l'âge de } 16 \text { ans ( } n=2669 \text { ) }\end{array}$ & $15,4 \%(412)$ & $14 \%(255)$ & $18,5 \%(157)$ & 0,003 \\
\hline $\begin{array}{l}\text { Drogues injectables utilisées les plus } \\
\text { couramment au cours des six mois } \\
\text { précédant l'entrevue }{ }^{1}\end{array}$ & $\begin{array}{l}64,3 \% \\
(1724) \\
47,2 \%\end{array}$ & & & \\
\hline Cocaïne & (1 265) & $66 \%(1206)$ & $60,8 \%(518)$ & 0,009 \\
\hline Hydromorphone & $47 \%$ (1 259) & $47,1 \%(861)$ & $47,4 \%(404)$ & 0,890 \\
\hline Morphine (non prescrite) & $37,7 \%$ & $45 \%(822)$ & $51,3 \%(437)$ & 0,002 \\
\hline Oxycodone & (1 012) & $36,8 \%(673)$ & $39,7 \%$ (339) & 0,143 \\
\hline Héroïne & $26,7 \%(716)$ & $27,5 \%(503)$ & $25 \%(213)$ & 0,170 \\
\hline \multicolumn{5}{|l|}{$\begin{array}{l}\text { Personne la plus souvent signalée avec } \\
\text { laquelle les participants ont utilisé des } \\
\text { drogues injectables dans les six mois } \\
\text { précédant l'entrevue }\end{array}$} \\
\hline Personne (c.-à-d. injecté seulement) & $59,3 \%$ & & & 0,145 \\
\hline $\begin{array}{r}\text { Amis ou personnes qu'ils connaissent } \\
\text { bien }\end{array}$ & & $\begin{array}{l}60,2 \% \\
(1101)\end{array}$ & $57,2 \%(487)$ & 0,020 \\
\hline Partenaires sexuels réguliers $^{3}$ & $(1354)$ & $49 \%(896)$ & $53,8 \%(458)$ & $<0,001$ \\
\hline Personnes qu'ils ne connaissaient pas & $31 \%(831)$ & $24,8 \%(453)$ & $44,4 \%(378)$ & 0,604 \\
\hline bien & $17,8 \%(478)$ & $18,1 \%(331)$ & $17,3 \%(147)$ & \\
\hline Membres de la famille & $10,6 \%(285)$ & $8 \%(147)$ & $16,2 \%(138)$ & \\
\hline $\begin{array}{l}\text { Proportion des participants ayant utilisé } \\
\text { des aiguilles ou des seringues stériles } \\
\text { lors de la dernière injection }(n=2663)^{4}\end{array}$ & $\begin{array}{l}94,5 \% \\
(2516)\end{array}$ & $\begin{array}{l}94,7 \% \\
(1721)\end{array}$ & $94 \%(795)$ & 0,433 \\
\hline $\begin{array}{l}\text { Proportion des participants ayant utilisé } \\
\text { une aiguille ou une seringue déjà utilisée } \\
\text { pour s'injecter des drogues au cours des } \\
\text { six mois précédant l'entrevue ( } n=2671 \text { ) }\end{array}$ & $15,5 \%(415)$ & $13,7 \%(249)$ & $19,6 \%(166)$ & $<0,001$ \\
\hline $\begin{array}{l}\text { Proportion des participants ayant } \\
\text { déclaré que leurs aiguilles ou seringues } \\
\text { avaient ensuite été utilisées par } \\
\text { quelqu'un d'autre à des fins d'injection } \\
\text { au cours des six mois précédant } \\
\text { l'entrevue ( } n=2646)\end{array}$ & $15,5 \%(409)$ & $12,7 \%$ (229) & $21,4 \%(180)$ & $<0,001$ \\
\hline $\begin{array}{l}\text { Proportion des participants ayant utilisé } \\
\text { un autre équipement d'injection déjà } \\
\text { utilisé pour s'injecter des drogues au } \\
\text { cours des six mois précédant l'entrevue } \\
(\mathrm{n}=2672)^{5}\end{array}$ & $34,5 \%(922)$ & $31,6 \%$ (576) & $40,9 \%(346)$ & $<0,001$ \\
\hline $\begin{array}{l}\text { Proportion des participants ayant } \\
\text { déclaré que leur autre équipement } \\
\text { d'injection déjà utilisé avait ensuite été }\end{array}$ & $33,1 \%(880)$ & $29,7 \%(540)$ & $40,3 \%(340)$ & $<0,001$ \\
\hline
\end{tabular}




\begin{tabular}{|r|c|c|c|c|}
\hline $\begin{array}{l}\text { utilisé par quelqu'un d'autre à des fins } \\
\text { d'injection au cours des six mois } \\
\text { précédant l'entrevue (n= 2 659) }\end{array}$ & & & \\
\hline $\begin{array}{r}\text { Endroit de l'injection le plus souvent } \\
\text { signalé au cours des six mois précédant } \\
\text { I'entrevue }^{6}\end{array}$ & & & & \\
Son appartement ou sa maison & $61,1 \%$ & $59 \%(1081)$ & $65,6 \%(561)$ & $<0,001$ \\
Chez des amis & $(1642)$ & $40,4 \%(740)$ & $45,7 \%(391)$ & 0,007 \\
Dans un lieu public & $42,1 \%$ & $41,8 \%(766)$ & $34,3 \%(293)$ & $<0,001$ \\
À I'hôtel, au motel & $(1131)$ & $14,6 \%(267)$ & $17,8 \%(152)$ & 0,080 \\
Dans un véhicule & & & \\
En cohabitation, en pension & $(19,4 \%$ & $14,4 \%(263)$ & $18,3 \%(156)$ & 0,009 \\
& $15,6 \%(419)$ & $8,9 \%(163)$ & $6,7 \%(57)$ & 0,052 \\
& $15,6 \%(419)$ & & & \\
& $8,2 \%(220)$ & & & \\
\hline
\end{tabular}

${ }^{1}$ Les participants ont indiqué toutes les drogues qu'ils s'étaient injectées à des fins non médicinales au cours des six mois précédant l'entrevue. Les drogues les plus souvent mentionnées parmi tous les participants sont présentées. Étant donné que les participants pouvaient choisir plus d'une réponse, le dénominateur total n'est pas présenté.

${ }^{2}$ Les participants ont indiqué tous les types de personnes avec lesquelles ils avaient utilisé des drogues injectables au cours des six mois précédant l'entrevue. Les personnes les plus fréquemment signalées sont présentées. Étant donné que les participants pouvaient choisir plus d'une réponse, le dénominateur total n'est pas présenté.

${ }^{3}$ Un partenaire sexuel régulier a été défini comme une personne avec laquelle le participant avait une relation et avec laquelle il était engagé sur le plan émotionnel.

${ }^{4}$ Cette mesure est aussi utilisée pour le calcul de l'indicateur du Rapport d'activité sur la riposte au sida dans le monde 2.3. (7)

${ }^{5}$ L'autre équipement d'injection usagé pouvait comprendre l'eau, les filtres, les réchauds, les cuillères, les garrots, les nœuds, les cotons-tiges et les acidifiants pour l'eau.

${ }^{6}$ Les participants ont déclaré tous les endroits où ils s'injectaient des drogues au cours des six mois précédant l'entrevue. Les endroits les plus souvent les plus souvent mentionnés parmi tous les participants sont présentés. Étant donné que les participants pouvaient choisir plus d'une réponse, le dénominateur total n'est pas présenté.

${ }^{7}$ Les lieux publics comprenaient les rues, les parcs, les squats, le métro, etc.

${ }^{8}$ Les véhicules comprenaient les voitures, les fourgonnettes, les véhicules récréatifs, etc.

Plusieurs différences ont été relevées entre les comportements d'usage et d'injection de drogues des hommes par rapport à ceux des femmes (tableau 3). Dans l'ensemble, 15,4\% de tous les participants ont déclaré qu'ils avaient utilisé des drogues injectables pour la première fois avant l'âge de 16 ans, avec une proportion significativement plus élevée de femmes que d'hommes indiquant une utilisation précoce de drogues injectables (18,5\% par rapport à $14 \%)$. Les participants ont déclaré une variété de substances qu'ils s'étaient injectées au cours des six mois précédant l'entrevue; la cocaïne était la plus couramment mentionnée parmi tous les participants $(64,3 \%)$. Une proportion significativement plus élevée d'hommes que de femmes a déclaré s'être injecté de la cocaïne (66\% par rapport à 60,8\%) alors qu'une proportion significativement plus élevée de femmes que d'hommes a déclaré s'être injecté de la morphine non prescrite $(51,3 \%$ par rapport à $45 \%)$.

En ce qui concerne les personnes avec lesquelles les participants utilisaient des drogues injectables au cours des six mois précédant l'entrevue, une proportion significativement plus élevée de femmes a déclaré avoir utilisé des drogues injectables avec des amis ou des personnes qu'elles connaissaient bien, des partenaires sexuels réguliers ou des membres de la famille. La proportion élevée de participants (59,3\%) ayant déclaré avoir utilisé des drogues injectables seuls est particulièrement préoccupante, car l'injection seule est un important facteur de risque de surdose et de décès (8).

Bien qu'une grande proportion $(94,5 \%)$ d'hommes et de femmes ait déclaré avoir utilisé une aiguille stérile lors de leur dernière injection, une proportion significativement plus élevée de femmes a déclaré des antécédents de comportements d'injection à risque élevé, y compris l'utilisation d'aiguilles, de seringues ou d'autre équipement d'injection contaminés, ainsi que le partage des aiguilles, des seringues ou d'autre équipement d'injection usagés à d'autres personnes. Ces résultats, combinés aux données provenant de la surveillance de routine nationale qui démontrent qu'une proportion plus élevée de femmes adultes par rapport à leurs homologues hommes est infectée par le VIH en raison de l'usage de substances psychoactives, semblent indiquer que les femmes qui utilisent des drogues injectables sont particulièrement vulnérables à l'infection au VIH (9). 
Les participants ont indiqué plusieurs emplacements où ils s'injectaient des drogues au cours des six mois précédant l'entrevue. L'emplacement le plus courant était leur propre appartement ou maison, et cet endroit a été signalé par une proportion significativement plus élevée de femmes que d'hommes (65,6\% par rapport à $59 \%)$. Une proportion significativement plus élevée de femmes que d'hommes a également déclaré avoir utilisé des drogues injectables chez un ami et dans un véhicule. En revanche, une proportion significativement plus élevée d'hommes que de femmes a déclaré avoir utilisé des drogues injectables dans un lieu public. Dans l'ensemble, 39,4\% de tous les participants ont déclaré avoir utilisé des drogues injectables dans un lieu public, ce qui est préoccupant étant donné que l'usage de drogues injectables dans un lieu public est associé à des pratiques d'injection à risque élevé et à un risque accru de transmission du VIH et d'autres pathogènes à diffusion hématogène (10).

Tableau 4 : Comportements sexuels à risque des participants à l'enquête de la phase 3 de I-Track

\begin{tabular}{|c|c|c|c|c|}
\hline Comportement sexuel & Total & Hommes & Femmes & Valeur $p$ \\
\hline $\begin{array}{l}\text { Proportion des participants ayant eu au } \\
\text { moins deux partenaires sexuels au cours } \\
\text { des six mois précédant l'entrevue } \\
(n=2676)^{1}\end{array}$ & $34,4 \%(920)$ & $31,3 \%(572)$ & $40,9 \%(348)$ & $<0,001$ \\
\hline $\begin{array}{l}\text { Proportion des participants qui avaient } \\
\text { utilisé un préservatif lors de leur dernière } \\
\text { relation sexuelle (parmi les participants } \\
\text { ayant déclaré avoir eu des relations } \\
\text { sexuelles au cours du mois dernier, } \\
n=2124)^{1}\end{array}$ & $36,6 \%(777)$ & $37,2 \%(505)$ & $35,4 \%(272)$ & 0,401 \\
\hline $\begin{array}{l}\text { Proportion de participants ayant eu un } \\
\text { partenaire-client dans les six mois } \\
\text { précédant l'entrevue ( } n=2687)^{1}\end{array}$ & $12,8 \%(343)$ & $4,7 \%(86)$ & $30,1 \%(257)$ & $<0,001$ \\
\hline $\begin{array}{l}\text { Proportion des participants qui avaient } \\
\text { utilisé un préservatif avec un partenaire- } \\
\text { client }(n=306)\end{array}$ & $77,1 \%(236)$ & $57,4 \%(35)$ & $82 \%(201)$ & $<0,001$ \\
\hline $\begin{array}{l}\text { Proportion des participants ayant déjà } \\
\text { reçu un diagnostic d'infection } \\
\text { transmissible sexuellement }(n=1732)^{2,3}\end{array}$ & $39,3 \%(680)$ & $32,7 \%(355)$ & $50,2 \%(325)$ & $<0,001$ \\
\hline
\end{tabular}

${ }^{1}$ Un partenaire-client est quelqu'un qui a échangé de l'argent, des drogues, des biens ou toute autre chose contre des relations sexuelles avec le participant.

${ }^{2}$ Définie comme le fait qu'un professionnel de la santé (p. ex. un médecin ou une infirmière) a déjà dit au participant qu'il avait la chlamydiose, la gonorrhée, le virus du papillome humain, l'herpès génital, l'herpès par voie orale ou une autre infection transmissible sexuellement.

${ }^{3}$ Les données sur les antécédents de diagnostics avec une infection transmissible sexuellement n'ont pas été recueillies dans le réseau SurvUDI.

Il a été démontré que l'utilisation de drogues avait une influence sur les comportements sexuels en augmentant la prise de risques (tableau 4). Par conséquent, la compréhension des comportements sexuels à risque élevé ( $p$. ex. l'utilisation irrégulière du préservatif, des partenaires sexuels multiples, le travail dans l'industrie du sexe) des utilisateurs de drogues injectables au Canada est donc de grande importance pour la santé publique (11). Parmi les participants à la phase 3 de I-Track ayant déclaré être sexuellement actifs, il existait des différences de comportement sexuel entre les femmes et les hommes. Une proportion significativement plus élevée de femmes que d'hommes a déclaré au moins deux partenaires sexuels au cours des six mois précédant l'entrevue. Parmi les participants ayant déclaré avoir eu des relations sexuelles au cours du mois précédant l'entrevue, l'utilisation du préservatif lors du dernier rapport sexuel était semblable entre les hommes et les femmes, quoique très faible parmi tous les participants $(36,6 \%)$. Il convient de noter que l'utilisation du condom au cours du dernier rapport sexuel a été mesurée pour tous les types de partenaire sexuel. En comparaison, l'utilisation du préservatif lors du dernier rapport sexuel avec un partenaire-client était considérablement plus élevée $(77,1 \%)$, et une proportion significativement plus élevée de femmes que d'hommes a déclaré ce comportement ( $82 \%$ par rapport à $57,4 \%$ ). Une proportion significativement plus élevée de femmes que d'hommes a déclaré avoir un partenaire-client au cours des six mois précédant l'entrevue (30,1\% par rapport à 4,7\%). Les antécédents d'un 
diagnostic d'infection transmissible sexuellement étaient significativement plus élevés chez les femmes que chez les hommes $(50,2 \%$ par rapport à $32,7 \%)$.

Tableau 5 : Dépistage et suivi du VIH et de I'hépatite C chez les participants à l'enquête de la phase 3 de I Track

\begin{tabular}{|c|c|c|c|c|}
\hline $\begin{array}{l}\text { Dépistage, soins, traitement et suivi du } \\
\text { VIH et de I'hépatite C }\end{array}$ & Total & Hommes & Femmes & Valeur $p$ \\
\hline \multicolumn{5}{|l|}{ VIH } \\
\hline $\begin{array}{l}\text { Proportion des participants ayant déjà } \\
\text { subi un test de dépistage du VIH } \\
\text { ( } n=2657)\end{array}$ & $92,9 \%(2468)$ & $\begin{array}{l}91,9 \% \\
(1668)\end{array}$ & $95,1 \%(800)$ & 0,002 \\
\hline $\begin{array}{l}\text { Proportion des participants ayant subi un } \\
\text { test de dépistage du VIH au cours des } \\
\text { deux années précédant l'entrevue (parmi } \\
\text { les participants ayant déclaré avoir eu des } \\
\text { résultats négatifs au test de dépistage du } \\
\text { VIH, } n=2010 \text { ) }\end{array}$ & $85 \%$ (1 709) & $\begin{array}{l}83,6 \% \\
(1133)\end{array}$ & $88,1 \%(576)$ & 0,008 \\
\hline $\begin{array}{l}\text { Proportion des participants ayant déclaré } \\
\text { qu'ils étaient suivis par un médecin en } \\
\text { raison de leur VIH au moment de } \\
\text { l'entrevue (parmi les participants ayant } \\
\text { indiqué être séropositifs pour le VIH, } \\
n=95)^{1}\end{array}$ & $95 \%(95)$ & $94,2 \%(49)$ & $95,8 \%(46)$ & $0,713^{2}$ \\
\hline $\begin{array}{l}\text { Proportion des participants ayant déjà } \\
\text { pris des médicaments prescrits pour le } \\
\text { VIH (parmi les participants ayant indiqué } \\
\text { être séropositifs pour le VIH, } n=77 \text { ) }\end{array}$ & $77 \%(77)$ & $80,8 \%(42)$ & $72,9 \%(35)$ & 0,351 \\
\hline $\begin{array}{l}\text { Proportion des participants qui prenaient } \\
\text { des médicaments prescrits pour le VIH au } \\
\text { moment de l'entrevue (parmi les } \\
\text { participants ayant déclaré être } \\
\text { séropositifs pour le VIH, } n=100 \text { ) }\end{array}$ & $66 \%(66)$ & $75 \%(39)$ & $56,3 \%(27)$ & 0,048 \\
\hline \multicolumn{5}{|l|}{ Hépatite C } \\
\hline $\begin{array}{l}\text { Proportion des participants ayant déjà } \\
\text { subi un test de dépistage de l'hépatite C } \\
(n=2646)\end{array}$ & $91,4 \%$ (2 417) & $\begin{array}{l}90,3 \% \\
(1625)\end{array}$ & $93,6 \%(792)$ & 0,004 \\
\hline $\begin{array}{l}\text { Proportion des participants ayant déclaré } \\
\text { qu'ils étaient suivis par un médecin en } \\
\text { raison de leur hépatite } C \text { au moment de } \\
\text { l'entrevue (parmi les participants ayant } \\
\text { indiqué être séropositifs pour l'hépatite C } \\
\text { au moment de l'entrevue, } n=1063)^{3}\end{array}$ & $48,4 \%(514)$ & $49,3 \%(358)$ & $46,3 \%(156)$ & 0,359 \\
\hline $\begin{array}{l}\text { Proportion des participants qui avaient } \\
\text { déjà pris des médicaments prescrits pour } \\
\text { I'hépatite } C \text { (parmi les participants ayant } \\
\text { déclaré être infectés par l'hépatite } C \text { au } \\
\text { moment de l'entrevue, } n=1060 \text { ) }\end{array}$ & $9,5 \%(101)$ & 10,8 \% (78) & $6,9 \%(23)$ & 0,045 \\
\hline
\end{tabular}




\section{Proportion des participants qui prenaient des médicaments prescrits pour l'hépatite C au moment de l'entrevue (parmi les participants ayant déclaré être infectés par l'hépatite $\mathrm{C}$ au moment de l'entrevue, $n=1$ 063) \\ Défini comme une seule visite (ou plus) chez un médecin pour le traitement du VIH, des services de counseling, des tests de suivi, etc., au cours des six mois précédant l'entrevue. \\ ${ }^{2}$ Il est à noter qu'en raison du faible nombre de résultats, les résultats doivent être interprétés avec prudence.

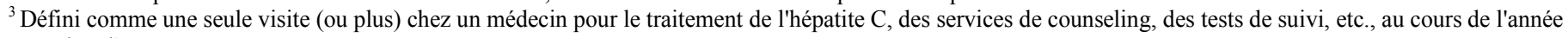 précédant l'entrevue.}

La plupart des participants ont indiqué qu'ils avaient déjà subi un test de dépistage du VIH et de l'hépatite $\mathrm{C}$ à un moment donné dans leur vie $(92,9 \%$ et $91,4 \%$, respectivement), et les antécédents de dépistage du VIH étaient significativement plus élevés chez les femmes pour les deux infections (tableau 5). Aucune différence significative n'a été trouvée entre les hommes et les femmes en ce qui a trait aux soins et aux traitements pour le VIH, à l'exception du fait qu'une proportion significativement plus élevée d'hommes s'étant déclarés séropositifs pour le VIH que de femmes ont déclaré qu'ils prenaient des médicaments prescrits pour le VIH au moment de l'entrevue (75 \% par rapport à $56,3 \%$, respectivement). Parmi les participants ayant déclaré être infectés par l'hépatite $C$ au moment de l'entrevue, de faibles proportions ont déclaré être suivies par un médecin et prendre des médicaments prescrits soit au moment de l'entrevue, soit par le passé. Aucune différence statistiquement importante n'a été trouvée entre les hommes et les femmes en ce qui a trait aux soins et au traitement de l'hépatite $C$, à l'exception du fait qu'une proportion significativement plus élevée d'hommes que de femmes ont déclaré qu'ils avaient, à un moment donné au cours de leur vie, pris des médicaments prescrits pour de l'hépatite $\mathrm{C}(10,8 \%$ par rapport à $6,9 \%$, respectivement). 
Tableau 6 : Recours à des services de santé et niveau de difficulté pour accéder à des aiguilles propres

\begin{tabular}{|c|c|c|c|c|}
\hline Recours et accès aux soins de santé & Total & Hommes & Femmes & Valeur $p$ \\
\hline \multicolumn{5}{|l|}{$\begin{array}{l}\text { Proportion des participants ayant déclaré } \\
\text { avoir utilisé les services de soins de santé } \\
\text { suivants au cours des } 12 \text { mois précédant } \\
\text { l'entrevue }\end{array}$} \\
\hline \multicolumn{5}{|l|}{$\begin{array}{l}\text { Service d'échange de seringues ou de } \\
\text { réduction des méfaits ( } n=1732 \text { ) }\end{array}$} \\
\hline Hôpitaux ( $n=1732$ ) & $89 \%$ (1 541) & $87,4 \%(948)$ & $91,7 \%(593)$ & 0,006 \\
\hline Centre d'accueil communautaire $(n=1733)$ & $59,4 \%(1029)$ & $57,9 \%(628)$ & $61,9 \%(401)$ & 0,105 \\
\hline Cliniques médicales ( $n=1730$ ) & $54,5 \%(945)$ & $55,2 \%(599)$ & $53,4 \%(346)$ & 0,464 \\
\hline Centres de santé communautaires & $47,1 \%(815)$ & $42,7 \%(462)$ & $54,6 \%(353)$ & $<0,001$ \\
\hline$(n=1735)$ & $44,9 \%(779)$ & $42,7 \%(464)$ & $48,5 \%(315)$ & 0,019 \\
\hline Établissement de désintoxication $(n=1731$ ) & $32,2 \%(557)$ & $31,9 \%(346)$ & $32,6 \%(211)$ & 0,765 \\
\hline \multicolumn{5}{|l|}{ Centre de toxicomanie et de santé mentale } \\
\hline$(n=1729)$ & $23,7 \%(409)$ & $21,6 \%(234)$ & $27,1 \%(175)$ & 0,009 \\
\hline Services adaptés aux cultures ( $n=1729$ ) & $10 \%(173)$ & $9,4 \%(102)$ & $11 \%(71)$ & 0,284 \\
\hline Centre de santé sexuelle $(n=1727)$ & $9,6 \%(165)$ & $7,3 \%(79)$ & $13,4 \%(86)$ & $<0,001$ \\
\hline \multicolumn{5}{|l|}{$\begin{array}{l}\text { Niveau de difficulté à accéder à des aiguilles } \\
\text { propres autodéclaré ( } n=2663 \text { ) }\end{array}$} \\
\hline $\begin{array}{r}\text { Très facile } \\
\text { Plus ou moins facile } \\
\text { Plus ou moins difficile } \\
\text { Très difficile }\end{array}$ & $\begin{array}{c}81 \%(2158) \\
15,5 \%(413) \\
3,1 \%(83) \\
0,3 \%(9)\end{array}$ & $\begin{array}{c}82,2 \% \\
(1493) \\
15 \%(272) \\
2,4 \%(44) \\
0,4 \%(8)\end{array}$ & $\begin{array}{c}78,6 \%(665) \\
16,7 \%(141) \\
4,6 \%(39) \\
0,1 \%(1)\end{array}$ & 0,006 \\
\hline
\end{tabular}

La prestation de services de santé et de soutien social pour les populations prioritaires, y compris les utilisateurs de drogues injectables, est un élément important de la stratégie de lutte contre le $\mathrm{VIH} / \mathrm{sida}$ et d'autres infections transmissibles sexuellement et par le sang au Canada. Par conséquent, la compréhension de l'utilisation des services de santé parmi les utilisateurs de drogues injectables au Canada est donc d'une importance capitale (12). Le recours aux services de soins de santé au cours des 12 mois précédant l'entrevue variait selon les services de santé en question; dans l'ensemble, les services d'échange de seringues ou de réduction des méfaits ont été le plus souvent utilisés chez tous les participants (89\%) (tableau 6). Le recours aux services de santé était plus élevé chez les femmes; une proportion significativement plus élevée de celles-ci a déclaré avoir eu accès à des services d'échange de seringues ou de réduction des méfaits, à des cliniques médicales, à des centres de santé communautaires, à des centres de toxicomanie et de santé mentale et à des centres de santé sexuelle. Une grande proportion des participants $(96,5 \%)$ a déclaré que leur niveau de difficulté à accéder à des aiguilles propres était soit très facile ou plus ou moins facile, et on a observé des différences significatives entre les hommes et les femmes.

\section{Conclusion}

Dans l'ensemble, la séroprévalence du VIH et l'exposition à vie à l'infection par l'hépatite C étaient élevées parmi les participants à la phase 3 de I-Track. Bien que de nombreux participants aient déclaré des pratiques d'injection et sexuelles sécuritaires ( $p$. ex. qu'elles s'abstiennent d'utiliser ou de partager du matériel contaminé, qu'elles utilisent un préservatif, etc.), une proportion élevée de participants a déclaré des comportements à risque associés à l'acquisition et à la transmission du VIH et d'autres infections transmissibles sexuellement et par le sang. Ces résultats laissent entendre 
que les utilisateurs de drogues injectables représentent encore un groupe à risque important dans l'épidémie de VIH au Canada, et mettent en évidence la nécessité de poursuivre les services de prévention et de traitement, ainsi que le dépistage régulier et intégré des utilisateurs de drogues injectables.

Ce système de surveillance améliorée est unique au Canada. Les données de I-Track sont collectées grâce à des enquêtes transversales périodiques menées dans des sites sentinelles partout au pays au moyen de stratégies d'échantillonnage et de recrutement cohérentes au fil du temps. Bien qu'il ne soit pas possible d'examiner directement la causalité, ces données de surveillance offrent une précieuse source d'information pour les services de prévention et de traitement et aux programmes à l'échelle locale, provinciale et nationale. I-Track utilise des méthodes d'échantillonnage de commodité non aléatoire pour surmonter certaines des difficultés à accéder à cette population difficile à atteindre. Par conséquent, ces résultats de surveillance peuvent ne pas être représentatifs de l'ensemble des utilisateurs de drogues injectables au Canada. À l'exception des résultats en laboratoire, les résultats de ce rapport sont fondés sur des données autodéclarées, qui peuvent être biaisées en raison de la désirabilité sociale et, par conséquent, il est possible que certains comportements à risque aient été surreprésentés ou sous-représentés.

\section{Remerciements}

La mise en place réussie de la phase 3 de I-Track a été rendue possible grâce à la collaboration des organisations communautaires, des autorités provinciales et locales en matière de santé, des chercheurs et de l'Agence de la santé publique du Canada. Nous tenons à reconnaître et à souligner les contributions des participants à l'enquête A-Track, des membres des équipes d'enquête, des lieux d'enquête, des chercheurs principaux du site sentinelle (Michel Alary, Patricia Bacon, Patricia Caetano, Russell Callaghan, Holly D'Angelo-Scott, Murray Fyfe, Jennifer Gratrix, Brendan Hanley, Maurice Hennink, Pascale Leclerc, Dar Malaviarachchi, Peggy Millson, Carole Morissette, William Osei, Emma Palmantier, Élise Roy, Rita Shahin, Ron Shore, Ameeta Singh, Susan Snelling, Nathan Teegee, Maureen Twigg, Bryna Warshawsky, Gaynor Watson-Creed, John Wylie), des cochercheurs et des collaborateurs. Nous tenons à souligner le soutien des laboratoires nationaux du VIH et de rétrovirologie (Paul Sandstrom, John Kim, Laurie Malloch, Richard Pilon) et de tous les autres membres de l'équipe de surveillance I-Track de l'Agence de la santé publique du Canada (Chris Archibald, Susanna Ogunnaike-Cooke, Jill Tarasuk, Rachel Bennett, Rachel MacLean, Stephen Cule, Qiong Li).

\section{Conflit d'intérêts}

Aucun

\section{Financement}

Ce travail a été appuyé par l'Agence de la santé publique du Canada.

\section{Références}

(1) Public Health Agency of Canada. Summary: Estimates of HIV prevalence and incidence in Canada, 2011. Ottawa: PHAC; 2012.

(2) Public Health Agency of Canada. Epidemiology of acute hepatitis C infection in Canada: Results from the enhanced hepatitis strain surveillance system (EHSSS). Ottawa: Centre for Communicable Diseases and Infection Control, PHAC; 2009.

(3) Public Health Agency of Canada. Summary of key findings from the I-Track pilot survey, 2010-2012. Ottawa: PHAC; 2014. http://www.phac-aspc.gc.ca/aids-sida/publication/index-fr.php\#surveillance

(4) Statistics Canada. 2011 national household survey: Aboriginal peoples in Canada: First Nations People, Métis and Inuit. The Daily, Statistics Canada catalogue no. 11-001-X. 2013 May 8. http://www.statcan.gc.ca/daily-quotidien/130508/dq130508a-fr.pdf

(5) Corneil TA, Kuyper LM, Shoveller J, Hogg RS, Li K, Spittal PM, et al. Unstable housing, associated risk behaviour and increased risk for HIV infection among injection drug users. Health Place. 2006; 12(1): 79-85.

(6) Public Health Agency of Canada. HIVIAIDS epi updates, July 2010. Ottawa: Centre for Communicable Diseases and Infection Control, PHAC; 2010.

(7) UNAIDS. Guidelines: Construction of core indicators for monitoring the 2011 Political Declaration on HIVIAIDS. Geneva: UNAIDS; 2011. 
http://www.unaids.org/en/media/unaids/contentassets/documents/document/2011/JC2215_Global_AIDS_Response_Progress_Re porting_en.pdf

(8) Strike C, Leonard L, Millson M, Anstice S, Berkeley N, Medd E. Ontario needle exchange programs: best practice recommendation. Toronto: Ontario Needle Exchange Coordinating Committee; 2006.

(9) Public Health Agency of Canada. At a glance - HIV and AIDS in Canada: surveillance report to December 31st, 2012. Ottawa: Centre for Communicable Diseases and Infection Control, PHAC; 2013.

(10) McKnight I, Maas B, Wood E, Tyndall MW, Small W, Lai C, et al. Factors associated with public injecting among users of Vancouver's supervised injection facility. American Journal of Drug \& Alcohol Abuse. 2007; 33(2): 319-325.

(11) Public Health Agency of Canada. HIV transmission risk: a summary of evidence. Ottawa: Centre for Communicable Diseases and Infection Control; 2013.

(12) Public Health Agency of Canada. Strengthening federal action in the Canadian response to HIVIAIDS2012 (consultation le 14 août 2012).

http://www.phac-aspc.gc.ca/aids-sida/fi-if/fa-if/3-fr.php 